\title{
Three-dimensional analysis of surface plasmon resonance modes on a gold nanorod
}

\author{
Yuan-Fong Chau, ${ }^{1}$ Min Wei Chen, ${ }^{2}$ and Din Ping Tsai ${ }^{2, *}$ \\ 'Department of Electronic Engineering, Ching Yun University, Jung Li, Taiwan \\ ${ }^{2}$ Department of Physics and Center for Nanostorage Research, National Taiwan University, Taipei, Taiwan \\ ${ }^{*}$ Corresponding author: dptsai@phys.ntu.edu.tw
}

Received 29 August 2008; revised 30 October 2008; accepted 3 November 2008; posted 10 November 2008 (Doc. ID 100695); published 16 January 2009

\begin{abstract}
We investigate the surface optical properties of a gold ( $\mathrm{Au}$ ) nanorod by using the finite-element method in a three-dimensional model. Results from the near-field optical images show spatially oscillatory patterns (nodal fields) on the surface, and these are attributable to plasmon-mode wave functions. We interpret these phenomena in terms of the electric field nodes on the surface of the rod, and the results show good agreement with our experimental observation in the optical images. () 2009 Optical Society of America

OCIS codes: $\quad 240.6680,260.3910$.
\end{abstract}

\section{Introduction}

Noble metal nanoparticles are known for their ability to support surface plasmon resonance (SPR), making them strong scatterers and absorbers of visible light with resonance peak wavelengths that are highly sensitive to the nanoparticle size, shape, and local environment[1]. These properties, together with advances in nanoparticle technologies, have led to applications in both scientific and technological research [2-4]. Besides fundamental significance, application of SPR has occurred in sensor technology, in the characterization of molecules at a dielectricmetal interface[5], in extremely sensitive surfaceenhanced Raman scattering [6], in plasmonic devices[7], etc.

Recently, subwavelength transport of surface plasmons (SPs) was demonstrated [8], and there has been intensive interest in the properties of SPs in confined metal structures [9-13]. As for the observation of electromagnetic $(\mathrm{E} \overline{\mathrm{M}})$ enhancement from nanostructures at scales below $100 \mathrm{~nm}$, explanations for the observed enhancement factors in surface-enhanced Raman scattering and the spatial distribution of the electric field near the metal nanorod, as well

0003-6935/09/030617-06\$15.00/0

(C) 2009 Optical Society of America as the role played by the plasmon modes in the rod, are still not fully understood. In our recent experiment [14] [the experimental setup as shown in Fig. 1(a)], we found that for the incident light polarized along the rod, the electric field intensity distribution is not uniform on the surface of the rod. The far-field intensity was measured by a charge-coupled device (CCD) as shown in Fig. 1(b), and nodal fields were observed on the surface of the Au nanorod.

Based on our previous work [15] and inspired by these interesting issues, we investigate in this paper numerically the interaction between incident wave and an $\mathrm{Au}$ nanorod in air using the finite-element method (FEM). Various effects due to the variation in aspect ratio of the rod and the polarization direction of the fields on the optical scattering and absorption cross sections, as well as the local induced charge densities at the surfaces of the rod, are discussed in detail. This analysis reveals several intriguing features of light propagation in an Au nanorod. In particular subwavelength resolution can be achieved on the $\mathrm{Au}$ nanorod when the localization of the light is within areas whose sizes is less than a wavelength. Simulation results show good agreement with our experimental optical images [14] and reveal characteristic features of plasmon modes in photoluminescence images, which can enable us to 
obtain knowledge of electric field distributions around the Au nanorod.

\section{Simulation Structure, Results, and Discussion}

The structure under study is an Au nanorod with diameter $D$ and length $L$ surrounded by air $\left(\epsilon_{\text {air }}=1\right)$ as shown in Figs. 1(c) and 1(d). The integrated boundary surface and the one for reflection for each calculation are given in the figure. The rod diameter is much shorter than the wavelength, while the length can be longer than the wavelength. We also define a coefficient $\eta=L / D$ to determine the aspect ratio between $L$ and $D$. In our numerical simulation, we use a three-dimensional (3D) calculation model that couples the incident light into an Au nanorod by using the FEM based on solving Maxwell's equations in the frequency domain [16] to calculate the near-field distribution, the far-field scattering cross section (SCS), and the absorption cross section (ACS). Throughout this paper, the Au permittivity data are obtained from Johnson and Christy [17] and fitted to the Drude model, with corrections to include the particle size effect [18]. Here, two different polarizations of incident light are considered, with the wave vector $k$ along the negative $x$-axis and the electric field $E$ parallel to the $x$-axis for the longitudinal mode, and with $k$ along the negative $y$-axis and $E$ perpendicular to the $x$-axis for the transverse mode, respectively.

To compare the behavior of total SCS with the backward SCS, an electromagnetic wave was incident on an Au nanorod with length $L=1 \mu \mathrm{m}$ and dia- meter $D=40 \mathrm{~nm}$. The far-field SCS of the total face and the reflection face as a function of wavelengths with $x$-polarized [Fig. 2(a)] and $y$-polarized [Fig. 2(b)] incident light, respectively, are shown in Fig. 2 . As clearly seen in Fig. 2(a) for $x$ polarization, strong excitation of the SPR peak occurs at $\lambda=630 \mathrm{~nm}$ (redlight region), and a second peak occurs at $\lambda=930 \mathrm{~nm}$ (infrared region). This indicates that the two wavelengths could be used to excite SPR for the longitudinal mode. In addition, a local minimum peak of SCS is at $\lambda=496 \mathrm{~nm}$, which can be assigned to the interband transition (because the interband damping has less effect on the near-field response). For the case of the transverse mode as shown in Fig. 2(b), only one SPR peak occurs at $\lambda=521 \mathrm{~nm}$, showing similar qualitative features for the total SCS and backward SCS. The optical images for the longitudinal mode (at $\lambda=630 \mathrm{~nm}$, red light) and the transverse mode (at $\lambda=530 \mathrm{~nm}$, green light) are also shown in the insets of Figs. 2(a) and 2(b), respectively.

It is well known that the physical mechanism of photoluminescence in a solid (for example, an Au nanorod) can be attributed to the electron-hole pair excitation. Relaxation of the initially excited electron and hole to new energy states occurs via a variety of scattering processes in the nanorod, and radiation is emitted when the electron and hole recombine. The incident light is scattered by the $\mathrm{Au}$ nanorod and couples to the SP mode. This interaction is thus proportional to $\sigma_{s} f_{m}$, where $\sigma_{s}$ is the SCS of the Au nanorod and $f_{m}$ is the fraction of scattered energy that

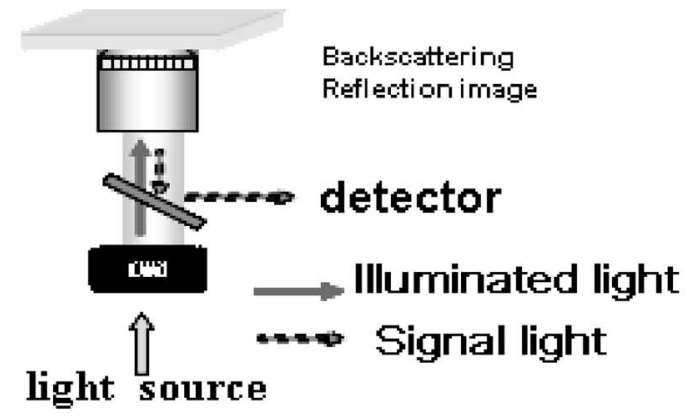

(a)

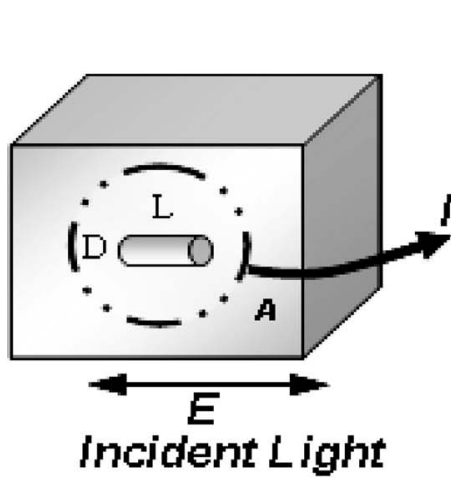

(c)

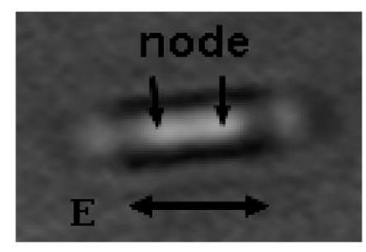

(b)

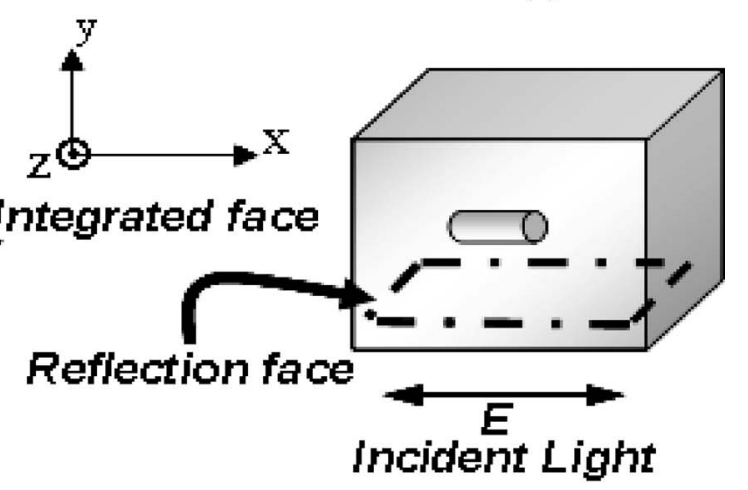

(d)

Fig. 1. Schematic of the experimental setup and simulation models: (a) experimental setup, (b) CCD image of a single Au nanorod, (c) integrated total faces of calculation model, and (d) reflection face of calculation model. 


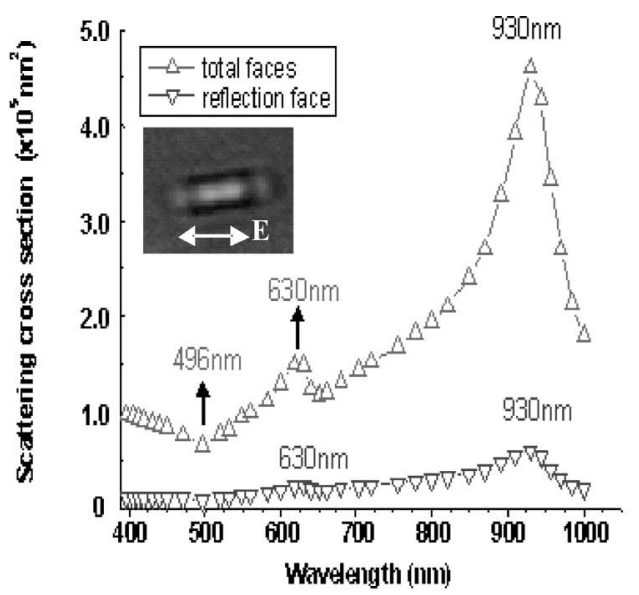

(a)

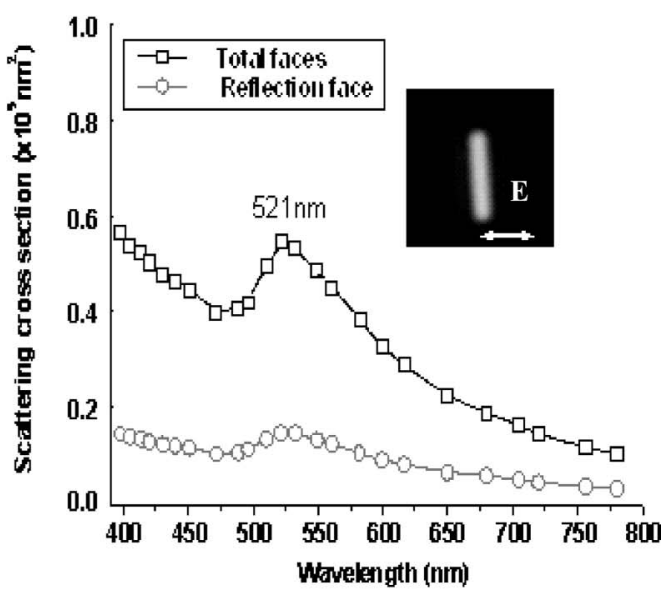

(b)

Fig. 2. Far-field SCS of total face and reflection face of an Au nanorod with $L=1 \mu \mathrm{m}$ and $D=40 \mathrm{~nm}$ as a function of wavelengths with (a) $x$-polarized and (b) $y$-polarized incident light, respectively. The optical images capture by CCD for longitudinal mode (at $\lambda=630 \mathrm{~nm}$, red light) and transverse mode (at $\lambda=530 \mathrm{~nm}$, green light) are also shown in the insets.

couples to the SP mode. The guided mode has a propagation length of $L$, hence the Au nanorod can act as a channel for the transformation of energy by means of SP. Ultimately, a fraction of this energy is scattered into air. To further understand the field pattern

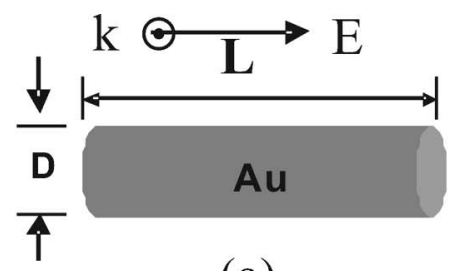

(a)
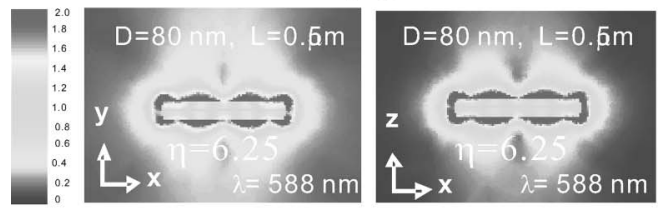

(b)
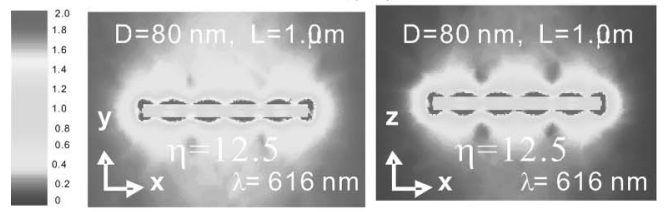

(c)
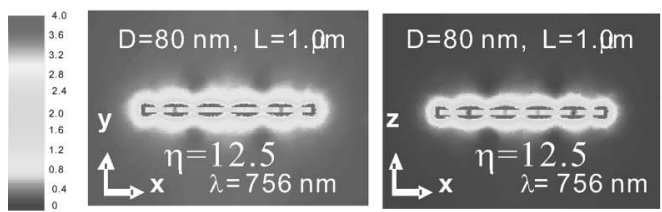

(d) produced inside and on the surface of the Au nanorod, we plotted the $x-y$ and $y-z$ cross-sectional planes of the scattered near-field amplitude $|E|$ distribution at SP modes for different $D$ and $L$ on the Au nanorod axis for longitudinal modes ( $x$ polarization). These

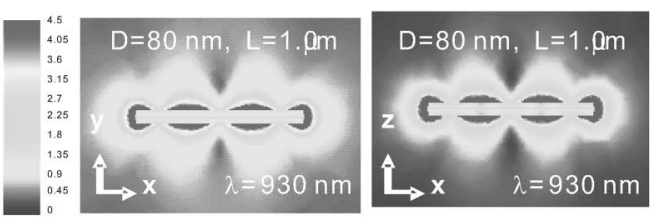

(e)

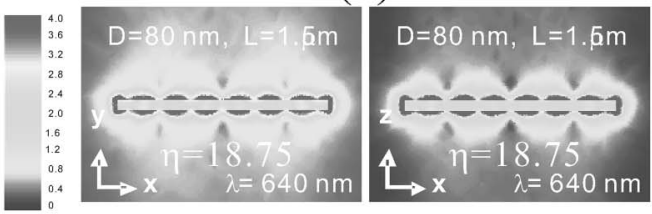

(f)

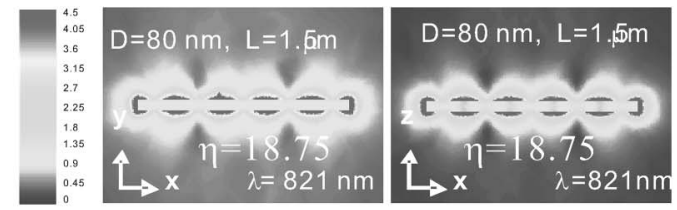

(g)

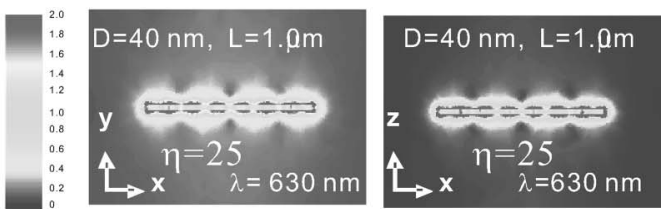

(h)

Fig. 3. $\quad x-y$ and $y-z$ cross-sectional planes of the scattered near-field amplitude $|E|$ distribution produced inside the single Au nanorod at SP modes for different $D$ and $L$ on the Au nanorod with $x$ polarization of incident light: (a) Schematic of the simulation model, (b)-(g) when the Au nanorod diameter $D$ is kept constant $(D=80 \mathrm{~nm})$ and the nanorod length $L$ is increased from $0.5 \mu \mathrm{m}$ to $1.5 \mu \mathrm{m}$, (h) aspect ratio $\eta=25(D=80 \mathrm{~nm}, L=0.5 \mu \mathrm{m})$. 


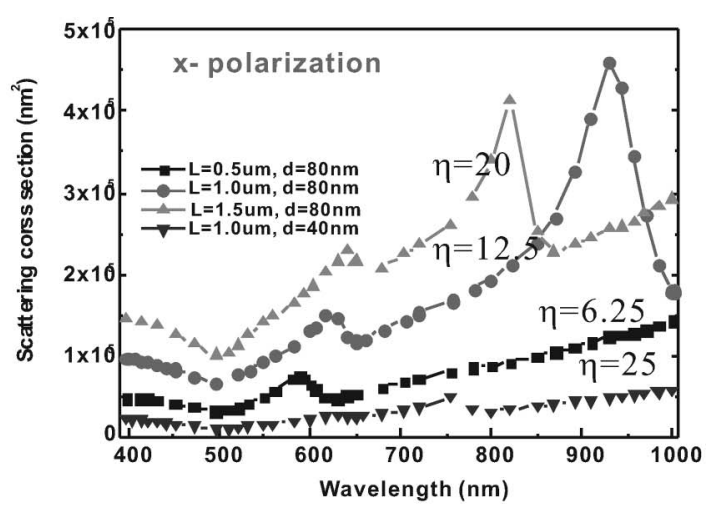

(a)

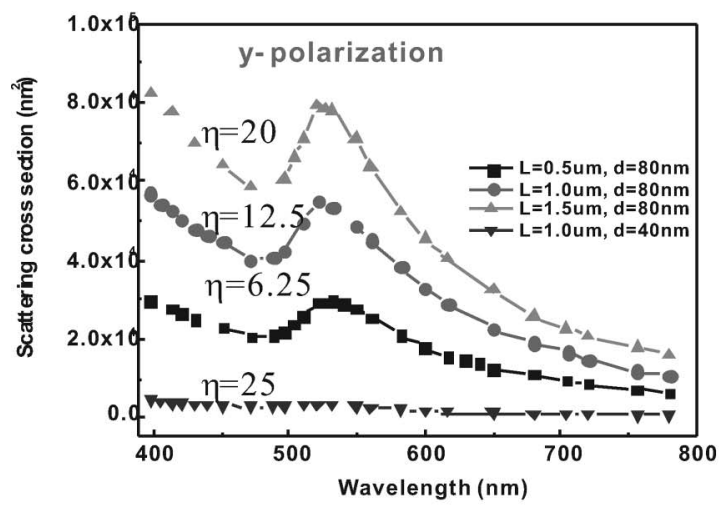

(b)

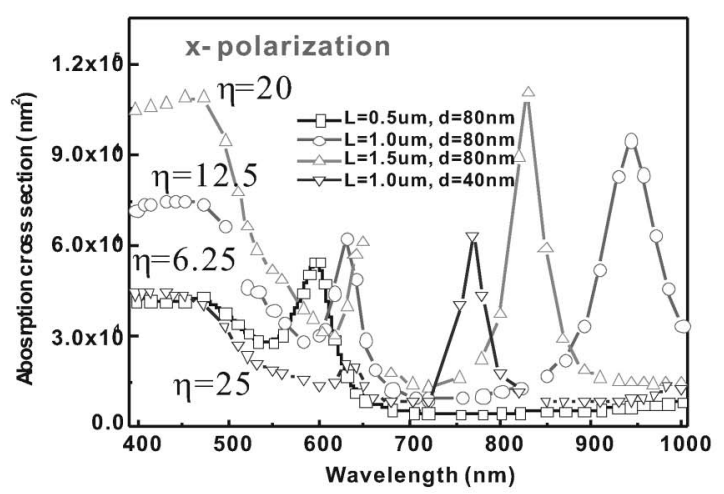

(c)

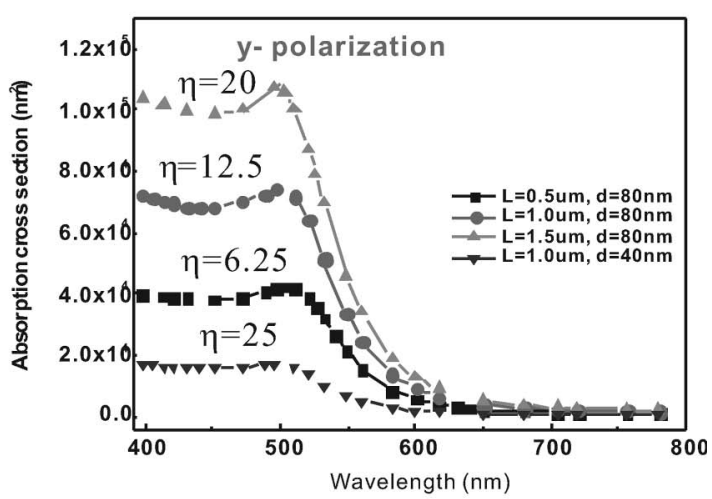

(d)

Fig. 4. Far-field scattering cross section [(a) and (b)] and absorption cross section [(c) and (d)] as a function of wavelengths for an EM plane wave incident on an Au nanorod with different aspect ratio for $x$-polarized [see (a) and (c)] and $y$-polarized [see (b) and (d)] incident light, respectively.

are displayed in Figs. 3(b)-3(h). The schematic of the simulation model is shown in Fig. 3(a). An interesting result, namely, some nodal fields, was found around the $\mathrm{Au}$ nanorod. When $D$ is kept constant $(D=80 \mathrm{~nm})$ and $L$ is increased from $0.5 \mathrm{~m}$ to $1.5 \mathrm{~m}$ [see Figs. 3(b)-3(g)], the nodal field pattern will be changed for various SPR wavelengths of the incident light. In our simulation, the zero-order mode is observed to have no nodal field oscillation along the $\mathrm{Au}$ nanorod. This mode has a finite charge and can be regarded as oscillations with an infinite wavelength - thus it cannot be excited by an external EM wave. The lowest mode with physical meaning is a dipolar mode with one nodal field around the Au nanorod, which has positive and negative charge accumulated at opposite ends of the Au nanorod. This mode can be excited by a field polarized along the $x$-axis and respond at a longer wavelength. Another mode can be identified by the number of nodal fields around the $\mathrm{Au}$ nanorod. It is worthwhile to note that here only even numbers of nodal fields can be found on the surface of the Au nanorod in our simulation. This result is very different from that obtained by means of the boundary element method [19], which includes odd and even modes simultaneously. The discrepancy between our 3D FEM simulation model and the boundary element method in
[18] can be attributed to the fact that the $x$ polarization incident field always piles up the positive and negative charge pairs along the propagation direction of the Au nanorod, and no net dipole exists in the charge distribution obtained in our simulation. Thus, only an even number of nodal fields can be found on the surface of the Au nanorod. For even-order modes, the wavelengths decrease as additional longitudinal nodes (positive and negative charge pairs) are added. It can be seen in Figs. 3(b)-3(h) for the case with $D=80 \mathrm{~nm}$ that the number of nodal fields around the Au nanorod for the longitudinal mode is dependent on the aspect ratio $\eta$, which corresponds to different eigenmodes and frequencies (wavelengths). As the aspect ratio $\eta$ increases, the nodal field number increases as $L$ increases. For the case of $\eta=25$ and $D=40 \mathrm{~nm}$ as shown in Fig. 3(h), a longer number of nodal field around the Au nanorod can be produced as $D$ decreases.

Figure 4 shows the far-field SCS [see Figs. 4(a) and 4(b)] and ACS [see Figs. 4(c) and 4(d)] as a function of wavelengths for an incident EM plane wave on an Au nanorod with different aspect ratios for $x$-polarized [see Figs. 4(a) and 4(c)] and $y$-polarized [see Figs. 4(b) and 4(d)] incident light, respectively. Four cases are displayed with the following parameters, i.e., $((D \mathrm{~nm}, L \mu \mathrm{m}, \eta)=(80,0.5,6.25),(80,1.0,12.5)$, 


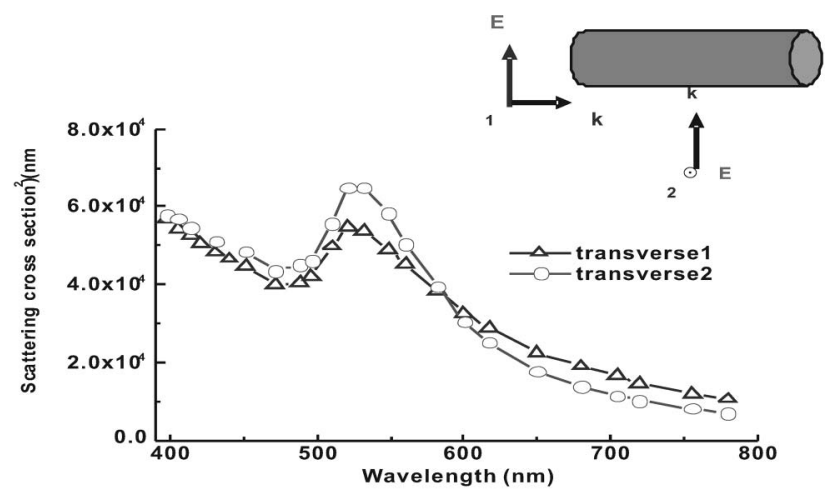

(a)

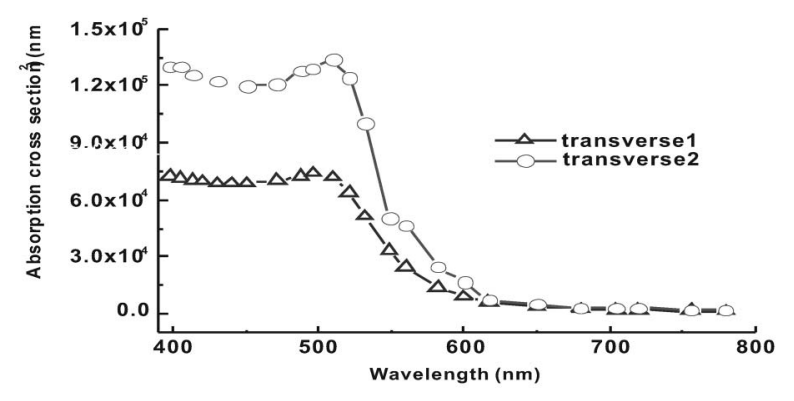

(b)

Fig. 5. Far-field SCS (a) and ACS (b) as a function of wavelengths for two different incident directions. The schematic of the simulation model is shown in the inset of the figure, where the case with transverse 1 has the electric $E$ parallel to the rod axis, and the case with transverse 2 has $E$ perpendicular to the rod axis.

$(80,1.5,20)$, and $(40,1.0,25)$. As can be seen in Figs. 4(a) and 4(c) for the case with $x$ polarization (longitudinal mode), the peak values of SCS are higher than those of ACS. A single peak is seen for the two cases: $((D \mathrm{~nm}, L \mu \mathrm{m}, \eta)=(80,0.5,6.25)$ and $(40,1.5$, 25 ). This implies that for small $D$ (for example, $D=40$, even if $\eta=25$ ) or small $\eta$ (for example, $\eta=$ 6.25 , even if $D=80 \mathrm{~nm}$ ) the long-wavelength peak cannot be excited. This long-wavelength peak originates from dipolar longitudinal charge oscillation [19] along the $\mathrm{Au}$ nanorod axis and is consistent with the mode charge distribution shown in Figs. $3(\mathrm{~b})-3(\mathrm{~h})$. As $L$ and $\eta$ increase while $\mathrm{D}$ is fixed at $8 \overline{0 \mathrm{~nm}}$ [for example $((D \mathrm{~nm}, L \mu \mathrm{m}, \eta)=(80$, $1.0,12.5),(80,1.5,20)]$, the first peak redshifts to the near infrared with increasing rod length $L$ and aspect ratio $\eta$. This is because the separation between the dipole charges inside the Au nanorod increases, thus reducing the restoring force that determines the oscillation frequency (or wavelength). Another interesting phenomenon emerges with the second peak being blueshifted. However a very different behavior is observed for $y$ polarization (transverse mode), as illustrated in Figs. 4(b) and 4(d). The peak values of the ACS [see Fig. 4(d)] are higher than those of SCS [see Fig. 4(b)]. When the aspect ratio $\eta$ increases, both the SCS and ACS peaks in the visible light region are blueshifted. From Fig. 4, one can predict that the longitudinal plasmon mode of the $\mathrm{Au}$ nanorod is close to red-light frequency, which is similar to the experimental result shown in the inset of Fig. 2(a) and is also similar to the transverse plasmon mode, which lies within the green light region [the experimental result shown in the inset of Fig. 2(b)].

In order to understand the transverse modes from different directions of incident light on the Au nanorod, in Fig. 5 we show the far-field SCS [Fig. 5(a)] and ACS [Fig. 5(b)] as a function of wavelengths for two different incident directions. The schematic of the simulation model and the direction of the incident wave are also shown in the inset of Fig. 5(a), where the wave vector $k$ is along the $y$-axis [perpendicular to the long axis ( $x$-axis) of the Au nanorod]. In Fig. 5 the case with transverse 1 has the electric field $\bar{E}$ parallel to the rod axis (i.e., along the $x$-axis), and the case with transverse 2 has $E$ perpendicular to the rod axis (i.e., along the $z$-axis). In comparison with the result shown in Fig. 5(a), it can be clearly observed in Fig. 5(b) that the discrepancy between

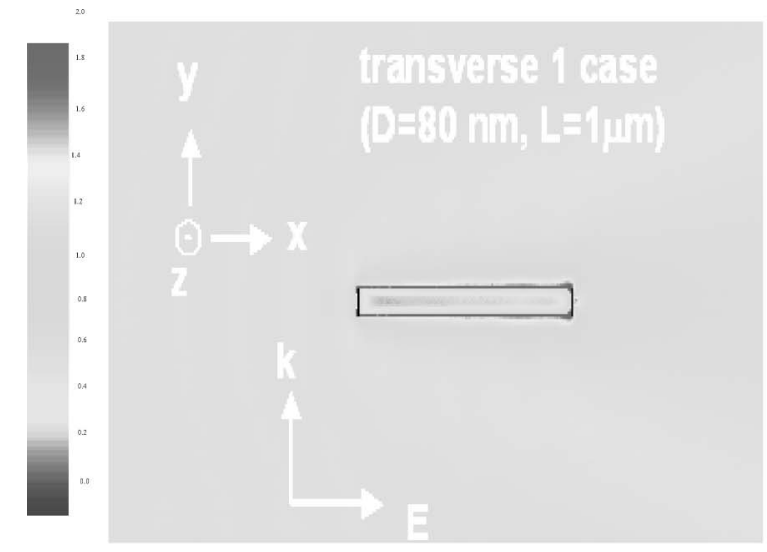

(a)

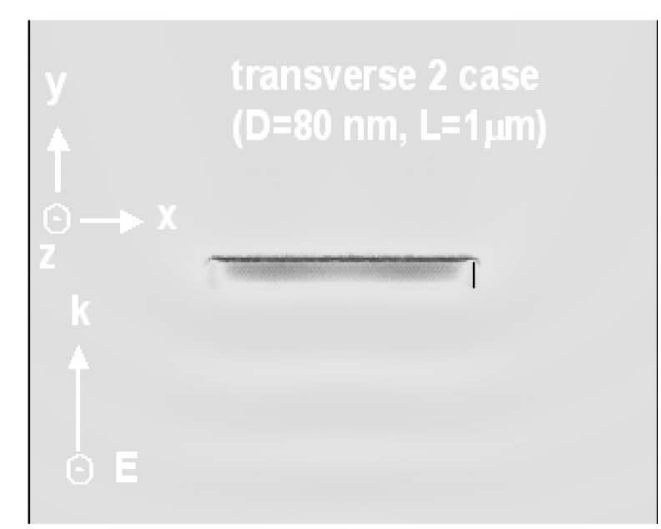

(b)

Fig. 6. $x-y$ cross-sectional plane of total field distribution of the (a) transverse 1 case and (b) transverse 2 case. 
the two transverse cases of ACS is distinct from that of SCS for the wavelength $\lambda$ less than $515 \mathrm{~nm}$, and the ACS declines dramatically as the wavelength of incident light becomes larger than $515 \mathrm{~nm}$. Figure $\underline{6}$ shows the $x-y$ cross-sectional plane of total field distribution for the transverse 1 case [see Fig. 5(a)] and transverse 2 case [see Fig. 5(b)], respectively. It can be clearly seen in Fig. 6(a) that the electric fields concentrate around the surface and the ends of the $\mathrm{Au}$ nanorod, and the propagating direction is along the long axis of the Au nanorod. On the contrary, the electric fields of transverse 2 case as shown in Fig. 6(b) are much weaker than those of transverse 1 case, and the propagation direction is along the short axis of the Au nanorod.

\section{Conclusion}

In conclusion, we have studied numerically the optical response of a single Au nanorod by using FEM to investigate their potential for the use of field-enhanced spectroscopy. The response of the single $\mathrm{Au}$ nanorod is sensitive to the polarization of the incident light and the rod aspect ratio. Polarization parallel to the rod axis ( $x$ polarization) provides the maximum enhancement, whereas polarization perpendicular to the rod axis ( $y$ polarization) generates weaker near fields. The number of nodal field on the surface of an Au nanorod can be carefully controlled for applications in field-enhanced spectroscopy. The tunable plasmon resonant scattering of Au nanorod provides an additional tool for the coupling of light into and out of optical waveguide structures. This property should lead to further applications in optical waveguide structures, waveguide-based photonic devices and other nanophotonic applications. The phenomena of nanometal are still not understood very well. Investigations are currently underway to explore further the near field of optical properties in coupled $\mathrm{Au}$ nanorod by experiments and simulation analysis. In the future, we will investigate different kinds of nanometal and their aspect ratios with different radii to study the size dependence as well as embedding of nanometal in different materials (oil, water) to investigate the substrate effect.

The authors are thankful for the financial support from the National Science Council of Taiwan under Grant numbers NSC 97-2120-M-002-013 and NSC 96-2112-M-231-001-MY3. We would also like to thank Hung Ji Huang for providing his experimental results and the National Center for HighPerformance Computing for support by providing their computing facility and software.

\section{References}

1. U. Kreibig and M. Vollmer, Optical Properties of Metal Clusters (Springer, 1995).

2. C. Oubre and P. Nordlander, "Optical properties of metallodielectric nanostructures calculated using the finite difference time domain method," J. Phys. Chem. B 108, 17740-17747 (2004).

3. S. Lal, S. L. Westcott, R. N. Taylor, J. B. Jackson, P. Nordlander, and N. J. Halas, "Light interaction between gold nanoshells plasmon resonance and planar optical waveguides," J. Phys. Chem. B 106, 5609-5612 (2002).

4. J. K. Lim, K. Imura, T. Nagahara, S. K. Kim, and H. Okamoto, "Imaging and dispersion relations of surface plasmon modes in silver nanorods by near-field spectroscopy," Chem. Phys. Lett. 412, 41-45 (2005).

5. A. J. Haes, S. Zou, G. C. Schatz, and R. P. Van Duyne, "A nanoscale optical biosensor: the long range distance dependence of the localized surface plasmon resonance of noble metal nanoparticles," J. Phys. Chem. B 108, 109-116 (2004).

6. S. Nie and S. R. Emory, "Probing single molecules and single nanoparticles by surface-enhanced Raman scattering," Science 275, 1102-1106 (1997).

7. W. L. Barnes, A. Dereux, and T. W. Ebbesen," Surface plasmon subwavelength optics," Nature 424, 824-830 (2003).

8. S. A. Maier, P. G. Kik, H. A. Atwater, S. Meltzer, E. Harel, B.E.Koel, and A.A. G. Requicha, "Local detection of electromagnetic energy transport below the diffraction limit in metal nanoparticle plasmon waveguides," Nat. Mater. 2, 229-232(2003).

9. P. Oleg, M. B. Varnavski, A. M. Mohamed, A. E. Mostafa, and G. Theodore, "Relative enhancement of ultrafast emission in gold nanorods," J. Phys. Chem. B 107, 3101-3104 (2003).

10. W. Rechberger, A. Hohenau, A. Leitner, J. R. Krenn, B. Lamprecht, and F. R. Aussenegg, "Optical properties of two interacting gold nanoparticles," Opt. Commun. 220, 137-141(2003).

11. J. J. Mock, S. J. Oldenburg, D. R. Smith, D. A. Schultz, and S. Schultz, "Composite plasmon resonant nanowires," Nano. Lett. 2, 465-469 (2002).

12. S. Lal, R. N. Taylor, J. B. Jackson, P. Nordlander, and N. J. Halas, "Light interaction between gold nanoshells plasmon resonance and planar optical waveguides," J. Phys. Chem. B 106, 5609-5612 (2002).

13. L. N. Colleen, K. G. Nathaniel, P. G. Glenn, T. Felicia, J. H. Naomi, and H. H. Jason, "Scattering spectra of single gold nanoshells," Nano. Lett. 4, 2355-2359 (2004).

14. H. J. Huang, C. P. Yu, H. C. Chang, K. P. Chiu, H. M. Chen, R. S. Liu, and D. P. Tsai, "Plasmonic optical properties of a single gold nano-rod," Opt. Express 15, 7132-7139 (2007).

15. Y.-F. Chau, D. P. Tsai, G.-W. Hu, L.-F. Shen, and T.-J. Yang, "Subwavelength optical imaging through a silver nanorod," Opt. Eng. 46, 039701 (2007).

16. http://www.ansoft.com/

17. P. B. Johnson and R. W. Christy, "Optical constants of the noble metals," Phys. Rev. B 6, 4370-4379 (1972).

18. T. Okamoto, in Near-Field Optics and Surface Plasmon Polaritons, S. Kawata, ed. (Springer, 2001), p. 99.

19. J. Aizpurua, Garnett W. Bryant, Lee J. Richter, F. J. Garcia de Abajo, Brian K. Kelley, and T. Mallouk, "Optical properties of coupled nanoscale metallic rods for field-enhanced spectroscopy," Phys. Rev. B 71, 235420 (2005). 